\title{
Association between EGFR exon 19 or exon 21 mutations and survival rates after first-line EGFR-TKI treatment in patients with non-small cell lung cancer
}

\author{
HAIYING JIANG, MEI ZHU, YANFANG LI and QIAN LI \\ Department of Oncology, Xuzhou Cancer Hospital, \\ Xuzhou, Jiangsu 221000, P.R. China
}

Received April 2, 2018; Accepted April 30, 2019

DOI: $10.3892 / \mathrm{mco} .2019 .1881$

\begin{abstract}
Epidermal growth factor receptor tyrosine kinase inhibitor (EGFR-TKI) is the first-line treatment for patients with advanced non-small-cell lung cancer (NSCLC) who have an EGFR mutation. However, little has been reported about the association between EGFR exon 19 deletions or an exon 21 mutation (specifically the L858R point mutation) and survival rates following first-line EGFR-TKI treatment in patients with NSCLC. As a retrospective study, 72 patients with stage IIIB/IV NSCLC carrying EGFR mutations (exon 19 deletions or an exon 21 mutation) were enrolled between 1 January 2008 and 31 December 2013, and all of the patients received first-line EGFR-TKI treatment. The associations between EGFR mutation status or clinical characteristics and response rate (ORR), progression-free survival (PFS) or overall survival (OS) were analyzed. Patients with exon 19 deletions (37 cases) had a higher ORR (75.7 vs. 51.4\%; $\mathrm{P}=0.032$ ), disease control rate (DCR; 89.2 vs. $68.6 \%$; $\mathrm{P}=0.031$ ), modified median $\mathrm{PFS}$ (13.2 vs. 10.8 months; $\mathrm{P}=0.030)$ and $\mathrm{OS}(30.2$ vs. 25.6 months; $\mathrm{P}=0.030)$ compared with those with an exon 21 mutation (35 cases). Cox multivariate analysis indicated that sex, histological type and smoking history were key factors that affected PFS and OS. Mutations status was associated with PFS, but not OS. Following EGFR-TKI therapy, a better ORR, DCR,
\end{abstract}

Correspondence to: Dr Haiying Jiang, Department of Oncology, Xuzhou Cancer Hospital, 131 Huancheng Road, Xuzhou, Jiangsu 221000, P.R. China

E-mail: jianghaiyinghu@sina.com

Abbreviations: EGFR-TKI, epidermal growth factor receptor tyrosine kinase inhibitor; NSCLC, non-small cell lung cancer; PFS, progression-free survival; OS, overall survival; EGFR, epidermal growth factor receptor; $\mathrm{CR}$, complete response; $\mathrm{PR}$, partial response; $\mathrm{SD}$, stable disease; $\mathrm{PD}$, progression disease; ORR, objective response rate; DCR, disease control rate

Key words: epidermal growth factor receptor, epidermal growth factor receptor tyrosine kinase inhibitor, non-small cell lung cancer, first-line
PFS and OS was observed in patients with EGFR deletions in exon 19 compared with those with an exon 21 mutation. The EGFR mutation status of patients with non-small cell lung cancer may therefore predict the efficacy and prognosis of EGFR-TKI.

\section{Introduction}

Lung cancer is the most common malignant tumor worldwide, with increasing annual incidence and mortality trends (1). Non-small-cell lung cancer (NSCLC) accounts for 80-85\% of all lung cancers (2). People diagnosed with NSCLC have a low 5-year survival rate $(<15 \%)(3)$ and $30-40 \%$ patients with locally advanced or metastatic cancer are not eligible for radical operation (4).

Epidermal growth factor receptor (EGFR) gene mutations frequently occur in exons 18-21, while deletions in exon 19 and a mutation in exon 21 (specifically the L858R point mutation) occur during EGFR-tyrosine kinase inhibitor (TKI) treatment (5). Among the non-selective Chinese patients with NSCLC, the total rate of EGFR mutations is $\sim 30 \%$ (6). In the patients with adenocarcinoma, the total rate of EGFR mutations is $50 \%$ and, in non-smoking patients with adenocarcinoma, it was 60-70\% (6). EGFR-TKI, a small molecule drug that is in competition with ATP, binds to the tyrosine kinase of EGFRs on the cell membrane. Consequently, EGFR-TKI can prevent tyrosine phosphorylation and inhibit a series of signaling pathways associated with the formation, proliferation and apoptosis of malignant cells, so as to inhibit the proliferation of tumor cells (7).

The existing literature revealed that the EGFR mutation status (deletions in exon 19 and an exon 21 mutation) was an important index for predicting the effectiveness of the EGFR-TKI in the treatment of NSCLC, which is the current standard first-line treatment $(8,9)$. However, there are only a small number of reports on the association between the two different mutation statuses and the response and survival rates following treatment (10).

The aim of the present study was to demonstrate that, NSCLC patients with EGFR exon deletions survive longer following EGFR-TKI treatment than those with exon 21 mutitions. 


\section{Materials and methods}

Eligibility criteria. The eligibility criteria were the following: i) Outpatients and inpatients diagnosed with advanced NSCLC (stage IIIB or IV) who had EGFR mutations (confirmed using second generation sequencing or the amplification refractory mutation system method) were enrolled in the Xuzhou Cancer Hospital (Xuzhou, China) between 1 January 2008 and 31 December 2013 [cancer classification was done according to the American Joint Committee on Cancer lung cancer staging criteria (Seventh Edition)] and were treated with the first-line EGFR-TKI treatment (11); ii) Aged between 18 and 75 years old; iii) A Eastern Cooperative Oncology Group performance status (PS) score of $\leq 2$ (12). All the patients were screened for EGFR gene expression using second generation sequencing or the amplification refractory mutation system method. The samples were analyzed using histological approach and iv) The presence of $\leq 1$ measurable lesion, according to the Response Evaluation Criteria in Solid Tumors (RECIST) 1.1 guidelines, which was defined as the target lesions measurement (13).

The detection of EGFR mutation by ARMS-PCR was performed as follows: Tissues were paraffin embedded and sliced into $10 \mu \mathrm{m}$ thick sections. Biopsy samples were stored for a maximum of 3 years. A microscope was utilized to observe the sections and to determine the tumor tissue content, the location of which was then marked. Tumor cells were then enriched to remove the effect of normal cells on the test results. Subsequently, commercial kits were utilized to extract human genomic DNA. Extracted DNA was then determined using a UV spectrophotometer. The optical density (OD) and concentration of DNA OD260/OD280 was required to be in the range of 1.8-2.0 and 3-300 ng/ $\mu 1$, respectively. Samples with a quality out of these ranges were discarded. Extracted DNA was then immediately tested or stored below $-20^{\circ} \mathrm{C}$ for $<6$ months. The negative and positive controls were then set. The positive control was required to exhibit a typical amplification curve. According to the $\mathrm{Ct}$ value of different fluorescent signal channels, the sample test results were analyzed to determine whether or not the EGFR mutation was present.

\section{Medical records and efficacy evaluation}

Treatment plan. A total of 72 patients with advanced NSCLC (IIIB/IV) who had EGFR mutations (exon 19 deletions or a mutation in exon 21) were subjected to first-line EGFR-TKI treatment. This treatment consisted $250 \mathrm{mg} /$ day gefitinib or $150 \mathrm{mg} /$ day erlotinib administered orally.

Efficacy evaluation. To evaluate the curative effect, the type of response [either complete response (CR), partial response (PR), stable disease (SD) or progression disease (PD)], the objective response rate (ORR) of CR and PR patients, and the disease control rate (DCR) of CR, PR and SD patients were determined 3 months after treatment. These criteria were examined according to the RECIST 1.1 guidelines. Progression-free survival (PFS) was defined as the duration between treatment initiation and the start of disease progression or mortality (all-cause). Overall survival (OS) was defined as the interval between the initiation of medication and mortality (all-cause) or the end of the follow-up period. Adverse drug reactions were evaluated according to the National Cancer Institute Common Terminology Criteria for Adverse Events (14). Other characteristics assessed were as follows: Sex, age, histological type, clinical stage, PS score, smoking history, name of EGFR-TKI administered, whether the patients received subsequent surgical treatment or chemotherapy. Non-smokers were defined as patient who smoked $\leq 100$ cigarettes during their lifetime. During the first two months of EGFR-TKI therapy, all patients underwent imaging examinations as detailed below, every $8 \pm 1$ weeks. Patients underwent chest, abdomen and pelvic computer tomography scans, and brain magnetic resonance imaging every 3 months until disease progression.

Follow-up. All the patients were regularly followed up by the telephone every 3 months. The last follow-up was performed 18 months after the last recruitment date.

Statistical analysis. SPSS 17.0 statistical software (SPSS, Inc., Chicago, IL, USA) was used for statistical analyses. Categorical variables were reported as numbers and percentages, and continuous variables were reported as the mean \pm standard deviation. Categorical variables were compared using a chi-square test. The association between EGFR mutation status, clinical characteristics and the effect of EGFR-TKI was tested by $\chi^{2}$; PFS and OS were analyzed with the log-rank test and Cox regression analysis. $\mathrm{P}<0.05$ was considered to indicate a statistically significant difference.

\section{Results}

Patients with deletions in exon 19 have similar clinical characteristics as patients with a mutation in exon 21. From a total of 72 patients, 37 cases were subjected to first-line oral gefitinib treatment and 35 to erlotinib; 20 cases were males and 52 cases were females (Table I). Among these cases, patients $\geq 70$ years old accounted for $\sim 51.4 \%$. In addition, 61 cases were diagnosed with adenocarcinoma and 11 with squamous cell carcinoma. A total of 33 cases were in the IIIB stage and 39 in the IV stage; 31 cases had PS scores of 0 or 1 and 41 had PS scores of 2 . There were a total of 15 smokers and 57 non-smokers. No statistical differences in these characteristics were identified between the exon 19 deletions and exon 21 mutation groups.

Patients with deletions in exon 19 exhibit an improved response and survival rate compared with patients that possess a mutation in exon 21 . The curative effect among 72 patients was evaluated; there were 2 cases of CR, 44 cases of PR, 11 cases of SD and 15 cases of PD, with an ORR of $44 \%$ and DCR of $72 \%$ (Table II). Furthermore, the patients with deletions in exon 19 of EGFR included 1 case of CR, 27 cases of PR, 5 cases of SD, 4 cases of PD and an ORR of $75.7 \%$, while patients with a mutation in exon 21 of EGFR included 1 case of CR, 17 cases of PR, 6 cases of SD, 11 cases of PD and an ORR of 51.4\%. The ORR of patients with deletions in exon 19 was significantly higher compared with the patients with a mutation in exon 21 $\left(\chi^{2}=4.583 ; \mathrm{P}=0.032\right)$. The patients with deletions in exon 19 had a DCR of $89.2 \%$, while those with a mutation in exon 21 had a DCR of $68.6 \%$. The DCR of patients with deletions in 
Table I. Clinical characteristics of 72 patients with advanced non-small cell lung cancer carrying EGFR mutation.

\begin{tabular}{|c|c|c|c|}
\hline Clinical characteristics & Exon 19 deletions $(\mathrm{n}=37)$ & An exon 21 mutation $(n=35)$ & P-value \\
\hline Sex & & & 0.884 \\
\hline Male & $10(27.0)$ & $10(28.6)$ & \\
\hline Female & $27(73.0)$ & $25(71.4)$ & \\
\hline Age (years) & & & 0.995 \\
\hline$<70$ & $18(48.6)$ & $17(48.6)$ & \\
\hline$\geq 70$ & $19(51.4)$ & $18(51.4)$ & \\
\hline Histological type & & & 0.820 \\
\hline Adenocacinoma & $31(83.8)$ & $30(85.7)$ & \\
\hline Squamous & $6(16.2)$ & $5(14.3)$ & \\
\hline Clinical stage & & & 0.984 \\
\hline IIIB & $17(45.9)$ & $16(45.7)$ & \\
\hline IV & $20(54.1)$ & $19(54.3)$ & \\
\hline PS score & & & 0.974 \\
\hline 0 or 1 & $16(43.2)$ & $15(42.9)$ & \\
\hline 2 & $21(56.8)$ & $20(57.1)$ & \\
\hline Smoking history & & & 0.866 \\
\hline Smoker & $8(21.6)$ & $7(20.0)$ & \\
\hline Non-smoker & $29(78.4)$ & $28(80.0)$ & \\
\hline EGFR-TKI & & & 0.642 \\
\hline Gefitinib & $20(54.1)$ & $17(48.6)$ & \\
\hline Erlotinib & $17(45.9)$ & $18(51.4)$ & \\
\hline Sequential surgery & & & 0.968 \\
\hline Surgery & 1 & 1 & \\
\hline Non-surgery & 36 & 34 & \\
\hline Sequential chemotherapy & & & 0.925 \\
\hline Chemotherapy & 5 & 32 & \\
\hline Non-chemotherapy & 5 & 30 & \\
\hline
\end{tabular}

Data are presented as $\mathrm{n}$ or $\mathrm{n}(\%)$. EGFR, epidermal growth factor receptor; TKI, tyrosine kinase inhibitor; PS, performance status

Table II. Efficacy of EGFR-TKI treatment.

\begin{tabular}{lccccccr}
\hline Group & CR & PR & SD & PD & ORR (\%) & P-value & DCR (\%) \\
\hline Exon 19 deletions & 1 & 27 & 5 & 4 & 75.7 & 0.032 & 89.2 \\
An exon 21 mutation & 1 & 17 & 6 & 11 & 51.4 & 68.6 \\
\hline
\end{tabular}

EGFR-TKI, epidermal growth factor receptor tyrosine kinase inhibitor; ORR, objective response rate; DCR, disease control rate; CR, complete response; $\mathrm{PR}$, partial response; $\mathrm{SD}$, stable disease; $\mathrm{PD}$, progression disease.

exon 19 was significantly higher compared with the patients with a mutation in exon $21\left(\chi^{2}=4.686 ; \mathrm{P}=0.031\right)$.

Following EGFR-TKI treatment, the modified median PFS in the patients with NSCLC who have deletions in exon 19 was 13.2 months, while it was 10.8 months in patients with a mutation in exon 21 (Fig. 1). The difference between the two groups' PFS was statistically significant $\left(\chi^{2}=4.700 ; \mathrm{P}=0.030\right)$. Furthermore, significant differences in the PFS were observed between the sexes, patients with adenocacinoma or squamous, smoking and non-smoking patients, and patients with mutations in exons 19 and 21 of EGFR (Table III). The results revealed that the median PFS in females $(\mathrm{P}=0.009)$, patients with deletions in exon $19(\mathrm{P}<0.001)$, patients with adenocarcinoma $(\mathrm{P}=0.004)$ and non-smoking patients $(\mathrm{P}=0.046)$ were significantly higher compared with the median PFS in males, patients with a mutation in exon 21, patients with squamous cell carcinoma and smoking patients, respectively. No statistically significant differences in PFS were observed in relation to the patients' age, tumor stage, PS score and whether the patient had received gefitinib or erlotinib. 


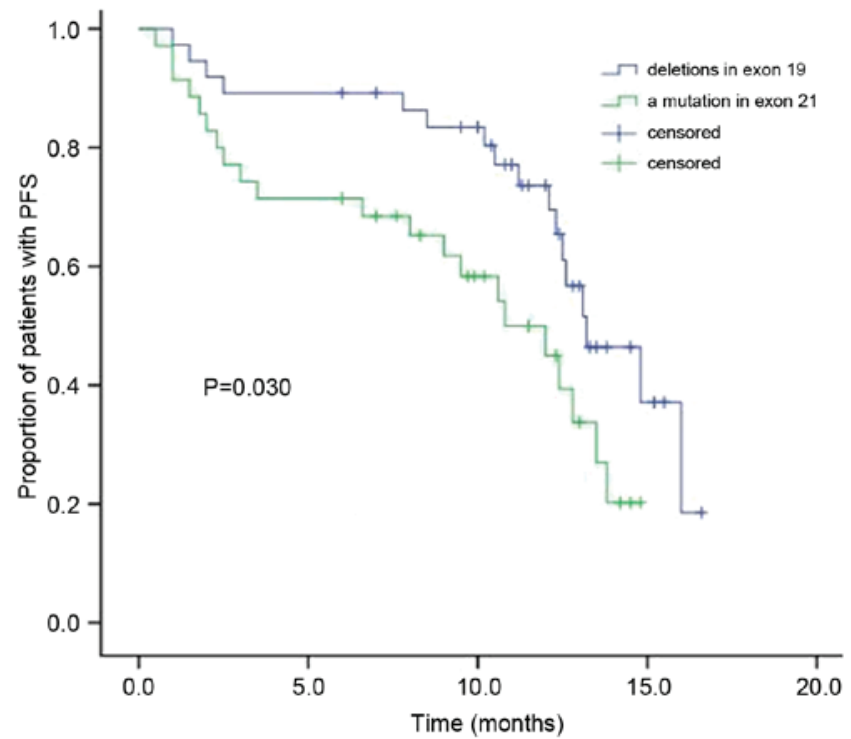

Figure 1. The Kaplan-Meier curve of PFS between the two groups. PFS, progression-free survival.

Following EGFR-TKI treatment, the median OS in the patients with NSCLC who had deletions in exon 19 was 30.2 months, while it was 25.6 months in patients with a mutation in exon 21 (Fig. 2). The difference between the two groups' OS was statistically significant $\left(\chi^{2}=4.700 ; \mathrm{P}=0.030\right)$. Cox multivariate analysis demonstrated that the median OS in females $(\mathrm{P}=0.018)$, patients with adenocarcinoma $(\mathrm{P}=0.009)$ and non-smoking patients $(\mathrm{P}=0.003)$ were significantly higher compared with the median OS of males, patients with squamous cell carcinoma and smoking patients, respectively (Table IV). No statistically significant differences in OS were observed in relation to the patients' age, tumor stage, PS score, whether the patient had received gefitinib or erlotinib and whether the patient had mutations in exon 19 or 21 of EGFR.

Patients with deletions in exon 19 are similarly affected by adverse reactions as patients with a mutation in exon 21 . The most common adverse reactions, which included rashes (48.6\%), diarrhea (26.4\%), coughs (2.8\%), stomatitis (4.17\%), anorexia (26.4\%), nausea (11.1\%) and vomiting (4.17\%), occurred in a similar frequency in patients with deletions in exon 19 compared with those with a mutation in exon 21 (Table V). The majority of these reactions were mild and moderate, while a small number were classified as third degree adverse reactions according to the National Cancer Institute Common Terminology Criteria for Adverse Events.

\section{Discussion}

The present study demonstrated that, following EGFR-TKI treatment for patients with NSCLC, the curative effect in patients with deletions in exon 19 was significantly higher compared with patients with a mutation in exon 21 (ORR, 75.7 vs. $51.4 \%$; DCR, 89.2 vs. $68.6 \%$ ). Furthermore, significantly higher modified median PFS (13.2 months) and median OS (30.2 months) times were observed in patients with deletions in exon 19 compared with patients with a mutation in

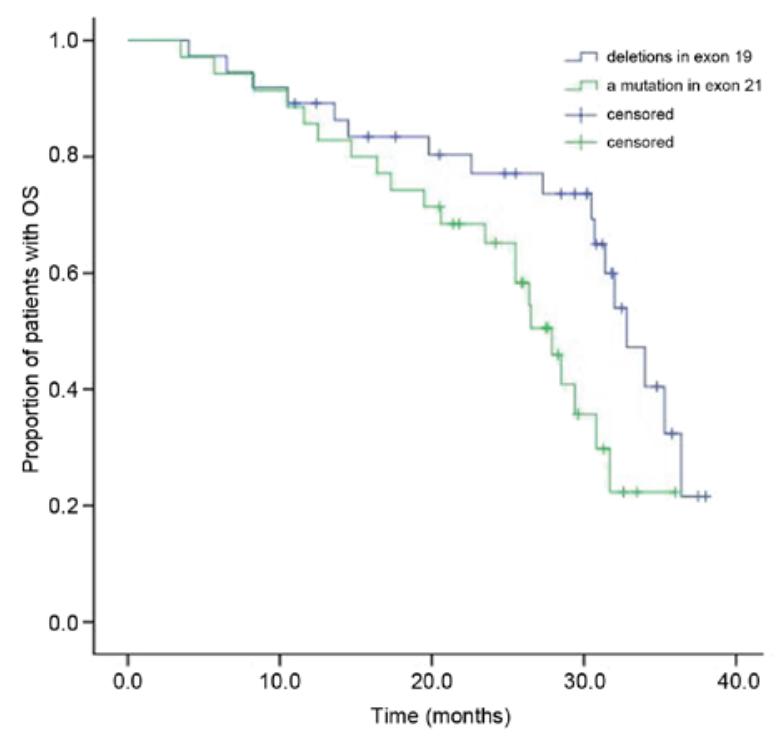

Figure 2. The Kaplan-Meier curve of OS between the two groups. OS, overall survival.

exon 21 (median PFS, 10.8 months; median OS, 25.6 months). Additionally, Cox multivariate analysis revealed that the PFS and OS were higher in female patients, patients with adenocarcinoma cell carcinoma and non-smoking patients, and PFS was higher in patients with deletions in exon 19. No significant differences in PFS and OS were observed in relation to patients' age, tumor stage, PS score and whether the patient had received gefitinib or erlotinib, and no significant difference in OS was identified in patients with exon mutations.

Eight randomized phase III trials (IPASS, Fist-SIGNAL, W3405, NEJ002, OPTIMAL, EURTAC, LUX-Lung 3, LUX-Lung 6) demonstrated the excellent efficacy of EGFR-TKI against NSCLC. Compared with platinum-based chemotherapy, which had a $30 \%$ remission rate and median PFS of 5-6 months, EGFR-TKI was determined to be a better first-line treatment for NSCLC patients with EGFR mutations, with a 70\% remission rate and median PFS of 10-11 months. Consequently, the consistency of EGFR-TKI demonstrated significant benefit in PFS and ORR as the first-line treatment in patients with EGFR mutations, thus it was established as a first-line treatment in these patients. According to some previous studies, patients with advanced NSCLC, an unknown EGFR mutation status or without any mutations, the first-line chemotherapy was revealed to be superior to first-line EGFR-TKI treatment (15-20). The LUX-Lung 3 clinical trail demonstrated that the median survival time for the patients with EGFR exon 19 deletions in the afatinib treatment group (33.3 months) was significantly longer compared with that of the chemotherapy treatment group (pemetrexed plus cisplatin; 21.1 months). For the patients with EGFR exon 21 mutations in the aforementioned trial, the survival time in the afatinib treatment group (27.6 months) was shorter compared with the chemotherapy treatment group (40.3 months). Furthermore, the LUX-Lung 6 clinical trail demonstrated that the median survival time for the patients with EGFR exon 19 deletions in the afatinib treatment group (31.4 months) was significantly longer compared with that of the chemotherapy treatment 
Table III. Cox multivariate analysis of PFS and clinical characteristics among 72 patients with advanced non-small cell lung cancer carrying EGFR mutation.

\begin{tabular}{|c|c|c|c|}
\hline Clinical characteristics & $\operatorname{Exp}(\mathrm{B})$ & $95 \% \mathrm{CI}$ & P-value \\
\hline Sex & & & 0.009 \\
\hline Male vs. female & 4.600 & $1.455-14.546$ & \\
\hline Age (years) & & & 0.468 \\
\hline$<70$ vs. $\geq 70$ & 1.378 & $0.579-3.280$ & \\
\hline Histological type & & & $<0.001$ \\
\hline Adenocacinoma vs. squamous & 0.011 & $0.001-0.119$ & \\
\hline Clinical stage & & & 0.340 \\
\hline IIIB vs. IV & 0.653 & $0.272-1.567$ & \\
\hline PS score & & & 0.748 \\
\hline 0 or 1 vs. 2 & 0.849 & $0.314-2.297$ & \\
\hline Smoking history & & & 0.004 \\
\hline Smoker vs. non-smoker & 9.742 & $2.026-46.672$ & \\
\hline EGFR-TKI & & & 0.789 \\
\hline Gefitinib vs. erlotinib & 0.887 & $0.368-2.136$ & \\
\hline Group & & & 0.046 \\
\hline Exon 19 deletions vs. an exon 21 mutation & 2.256 & $1.013-5.025$ & \\
\hline
\end{tabular}

EGFR, epidermal growth factor receptor; TKI, tyrosine kinase inhibitor; PS, performance status; PFS, progression-free survival; CI, confidence interval; Exp (B), exponentiation of the B coefficient, an odds ratio.

Table IV. Cox multivariate analysis of OS and clinical characteristics among 72 patients with advanced non-small cell lung cancer carrying EGFR mutation.

\begin{tabular}{|c|c|c|c|}
\hline Clinical characteristics & $\operatorname{Exp}(\mathrm{B})$ & $95 \% \mathrm{CI}$ & P-value \\
\hline Sex & & & 0.018 \\
\hline Male vs. female & 3.152 & $1.220-8.141$ & \\
\hline Age (year) & & & 0.411 \\
\hline$<70$ vs. $\geq 70$ & 1.455 & $0.595-3.559$ & \\
\hline Histological type & & & 0.009 \\
\hline Adenocacinoma vs. squamous & 0.059 & $0.007-0.494$ & \\
\hline Clinical stage & & & 0.764 \\
\hline IIIB vs. IV & 0.875 & $0.366-2.094$ & \\
\hline PS score & & & 0.368 \\
\hline 0 or 1 vs. 2 & 0.646 & $0.250-1.671$ & \\
\hline Smoking history & & & 0.003 \\
\hline Smoker vs. non-smoker & 8.322 & $2.061-33.614$ & \\
\hline EGFR-TKI & & & 0.787 \\
\hline Gefitinib vs. erlotinib & 1.072 & $0.649-1.771$ & \\
\hline Group & & & 0.426 \\
\hline Exon 19 deletions vs. an exon 21 mutation & 1.388 & $0.618-3.117$ & \\
\hline
\end{tabular}

EGFR, epidermal growth factor receptor; TKI, tyrosine kinase inhibitor; PS, performance status; OS, overall survival; CI, confidence interval; $\operatorname{Exp}(\mathrm{B})$, exponentiation of the $\mathrm{B}$ coefficient, an odds ratio.

group (18.4 months). As for the patients with EGFR exon 21 mutations, the survival time in the afatinib treatment group
(19.6 months) was shorter compared with that of the chemotherapy treatment group (24.3 months). The two clinical trials 
Table V. Adverse reactions among 72 patients with advanced non-small cell lung cancer and epidermal growth factor receptor mutations.

\begin{tabular}{lcccr}
\hline Adverse reaction & Exon 19 deletions $(\mathrm{n}=37)$ & An exon 21 mutation $(\mathrm{n}=35)$ & $\chi^{2}$ & P-value \\
\hline Rash & $18(48.6)$ & $17(48.6)$ & $<0.001$ & 0.995 \\
Degree III & 1 & 1 & & \\
Degree IV & 0 & 0 & & 0.016 \\
Diarrhea & $10(27.0)$ & $9(25.7)$ & & 0.007 \\
Degree III & 0 & 1 & & 0.934 \\
Degree IV & 0 & 0 & & \\
Nausea & $4(10.8)$ & $4(11.4)$ & & 0.292 \\
Degree III & 0 & 0 & & \\
Degree IV & 0 & 0 & 0.589 \\
Vomiting & $2(5.4)$ & $1(2.9)$ & & 0.954 \\
Degree III & 0 & 0 & & \\
Degree IV & 0 & 0 & & \\
Fatigue & $2(5.4)$ & $2(5.7)$ & 0.049 & 0.523 \\
Degree III & 0 & 0 & 0.292 & 0.589 \\
Degree IV & 0 & $2(5.7)$ & 0.167 & 0.683 \\
Cough & $1(2.7)$ & $1(2.9)$ & & \\
Stomatitis & $2(5.4)$ & $10(28.6)$ & 0 & \\
Anorexia & $9(24.3)$ & 0 & &
\end{tabular}

Data are presented as $\mathrm{n}$ or $\mathrm{n}(\%)$.

demonstrated that afatinib could significantly prolong the survival time for patients with NSCLC and EGFR deletions of exon 19, but it could not prolong the survival time for the patients with exon 21 mutations $(21,22)$.

Banno et al (23) confirmed that patients with NSCLC and EGFR exon 19 deletions had a distinct advantage following the treatment of afatinib compared with the patients with EGFR exon 21 mutations. The report from the NEJ002 study revealed that the patients with NSCLC and 21 exon mutations exhibited a relatively poor response to gefitinib, while the patients with NSCLC and exon 19 deletions had a greater response following gefitinib treatment (24). A meta-analysis revealed that, following EGFR-TKI as the first-line treatment for patients with NSCLC, the PFS for the patients with exon 19 deletions were markedly prolonged compared with patients with EGFR exon 21 mutations, however the study did not analyze OS (25). Additionally, a systematic review and meta-analysis revealed that in the patients with NSCLC and exon 19 deletions, OS was significantly improved following the EGFR-TKI treatment compared with those who had chemotherapy, while the patients with EGFR exon 21 mutations had no OS benefits with different treatments (5). Another study demonstrated that among the patients with advanced NSCLC that were treated with first-line TKI therapy, those with exon 19 deletions had longer PFS compared with those with exon 21 mutations (25). However, a retrospective clinical study conducted by Igawa et al (26) determined that no significant difference in ORR, PFS and OS was identified between patients with exon 19 deletions and patients with exon 21 mutations treated with gefitinib. In conclusion, the efficacy and survival rate of the afatinib treatment in patients with NSCLC and exon 19 deletions was better compared with those with exon 21 mutations. Only a small number of studies contradict the aforementioned studies regarding the comparison of the efficacy and survival rate between the two different mutations following EGFR-TKI treatment (10).

The studies described in the literature review had similar results to those obtained in the current study, indicating that following the administration of EGFR-TKI as the first-line treatment, patients with advanced NSCLC and deletions in exon 19 have greater ORRs, DCRs, PFS and OS compared with patients with mutations in exon 21. These rates and survival times were markedly improved in female patients, non-smoking and adenocarcinoma patients. Additionally, the two groups manifested predominantly mild side effects.

Therefore, the EGFR mutation status can be used as a predictive factor for the efficacy of EGFR-TKI as the first-line treatment among patients with advanced NSCLC. Furthermore, the patients with deletions in exon 19 of EGFR have significantly better outcomes in terms of response and survival rates compared with patients with a mutation in exon 21 of EGFR. However, due to the fact that the number of cases in the current study was quite small, further prospective and multicenter studies are required. The EGFR mutation status of patients with non-small cell lung cancer may predict the efficacy and prognosis of EGFR-TKI. 


\section{Acknowledgements}

Not applicable.

\section{Funding}

No funding received.

\section{Availability of data and materials}

The datasets used and/or analyzed during the present study are available from the corresponding author on reasonable request.

\section{Authors' contributions}

HJ performed the studies, participated in collecting data and drafted the manuscript. MZ and YF performed the statistical analysis and participated in its design. QL helped to draft the manuscript. All authors read and approved the final manuscript.

\section{Ethics approval and consent to participate}

The present study was approved by the Ethics Committees of Xuzhou Cancer Hospital (Xuzhou, China) and all patients provided written informed consent.

\section{Patient consent for publication}

Not applicable.

\section{Competing interests}

The authors declare that they have no competing interests.

\section{References}

1. Chang JS, Chen LT, Shan YS, Lin SF, Hsiao SY, Tsai CR, Yu SJ and Tsai HJ: Comprehensive analysis of the incidence and survival patterns of lung cancer by histologies, including rare subtypes, in the era of molecular medicine and targeted therapy: A nation-wide cancer registry-based study from Taiwan. Medicine (Baltimore) 94: e969, 2015.

2. Cho JH: Immunotherapy for non-small-cell lung cancer: Current status and future obstacles. Immune Netw 17: 378-391, 2017.

3. Jemal A, Bray F, Center MM, Ferlay J, Ward E and Forman D: Global cancer statistics. CA Cancer J Clin 61: 69-90, 2011.

4. Kiura K, Ueoka H, Segawa Y, Tabata M, Kamei H, Takigawa N, Hiraki S, Watanabe Y, Bessho A, Eguchi K, et al: Phase I/II study of docetaxel and cisplatin with concurrent thoracic radiation therapy for locally advanced non-small-cell lung cancer. $\mathrm{Br}$ J Cancer 89: 795-802, 2003.

5. Kuan FC, Kuo LT, Chen MC, Yang CT, Shi CS, Teng D and Lee KD: Overall survival benefits of first-line EGFR tyrosine kinase inhibitors in EGFR-mutated non-small-cell lung cancers: A systematic review and meta-analysis. Br J Cancer 113: $1519-1528,2015$

6. Stinchcombe TE: Targeted therapies for lung cancer. Cancer Treat Res 170: 165-182, 2016

7. Lemmon MA and Schlessinger J: Cell signaling by receptor tyrosine kinases. Cell 141: 1117-1134, 2010.

8. Zhou C, Wu YL, Chen G, Feng J, Liu XQ, Wang C, Zhang S, Wang J, Zhou S, Ren S, et al: Erlotinib versus chemotherapy as first-line treatment for patients with advanced EGFR mutation-positive non-small-cell lung cancer (OPTIMAL, CTONG-0802): A multicentre, open-label, randomised, phase 3 study. Lancet Oncol 12: 735-742, 2011.
9. Thatcher N, Chang A, Parikh P, Rodrigues Pereira J, Ciuleanu T, von Pawel J, Thongprasert S, Tan EH, Pemberton K, Archer V and Carroll K: Gefitinib plus best supportive care in previously treated patients with refractory advanced non-small-cell Lung Cancer: Results from a randomised, placebo-controlled, multicentre study (Iressa Survival Evaluation in Lung Cancer). Lancet 366: 1527-1537, 2005.

10. Tiseo M, Rossi G, Capelletti M, Sartori G, Spiritelli E, Marchioni A, Bozzetti C, De Palma G, Lagrasta C, Campanini N, et al: Predictors of gefitinib outcomes in advanced non-small lung cancer (NSCLC): Study of a comprehensive panel of molecular markers. Lung Canser 67: 355-360, 2010.

11. Rusch VW, Rice TW, Crowley J, Blackstone EH, Rami-Porta R and Goldstraw P: The seventh edition of the american joint committee on cancer/international union against cancer staging manuals: The new era of data-driven revisions. J Thorac Cardiovasc Surg 139: 819-821, 2010.

12. Oken MM, Creech RH, Tormey DC, Horton J, Davis TE, McFadden ET and Carbone PP: Toxicity and response criteria of the eastern cooperative oncology group. Am J Clin Oncol 5: 649-655, 1982.

13. Eisenhauer EA, Therasse P, Bogaerts J, Schwartz LH, Sargent D, Ford R, Dancey J, Arbuck S, Gwyther S, Mooney M, et al: New response evaluation criteria in solid tumors: Recist guideline (version 1.1). Eur J Cancer 47: 228-247, 2009.

14. Lakshman A, Modi M, Prakash G, Malhotra P, Khadwal A, Jain S, Kumari S, Varma N and Varma S: Evaluation of bortezomib-induced neuropathy using total neuropathy score (Reduced and Clinical Versions) and NCI CTCAE v4.0 in newly diagnosed patients with multiple myeloma receiving bortezomib-based induction. Clin Lymphoma Myeloma Leuk 17: 513-519, 2017.

15. Inoue A, Kobayashi K, Maemondo M, Sugawara S, Oizumi S, Isobe $\mathrm{H}$, Gemma A, Harada M, Yoshizawa H, Kinoshita I, et al: North-East Japan Study Group: Updated overall survival results from a randomized phase III trial comparing gefitinib with carboplatin-paclitaxel for chemo-naïve non-small cell lung cancer with sensitive EGFR gene mutations (NEJ002). Ann Oncol 24: 54-59, 2013.

16. Chen G, Feng J, Zhou C, Wu YL, Liu XQ, Wang C, Zhang S, Wang J, Zhou S, Ren S, et al: Quality of life (QoL) analyses from OPTIMAL (CTONG-0802), a phase III, randomised, open-label study of first-line erlotinib versus chemotherapy in patients with advanced EGFR mutation-positive non-small-cell lung cancer (NSCLC). Ann Oncol 24: 1615-1622, 2013.

17. Mitsudomi T, Morita S, Yatabe Y, Negoro S, Okamoto I, Tsurutani J, Seto T, Satouchi M, Tada H, Hirashima T, et al: West Japan Oncology Group: Gefitinib versus cisplatin plus docetaxel in patients with non-small-cell lung cancer harbouring mutations of the epidermal growth factor receptor (WJTOG3405): An open label, randomised phase 3 trial. Lancet Oncol 11: 121-128, 2010.

18. Rosell R, Carcereny E, Gervais R, Vergnenegre A, Massuti B, Felip E, Palmero R, Garcia-Gomez R, Pallares C, Sanchez JM, et al: Spanish lung cancer group in collaboration with groupe français de pneumo-cancérologie and associazione italiana oncologia toracica: Erlotinib versus standard chemotherapy as first-line treatment for European patients with advanced EGFR mutation-positive non-small-cell lung cancer (EURTAC): A multicentre, open-label, randomised phase 3 trial. Lancet Oncol 13: 239-246, 2012.

19. Fukuoka M, Wu YL, Thongprasert S, Sunpaweravong P, Leong SS, Sriuranpong V, Chao TY, Nakagawa K, Chu DT, Saijo N, et al: Biomarker analyses and final overall survival results from a phase III, randomized, open-label, first-line study of gefitinib versus carboplatin/paclitaxel in clinically selected patients with advanced non-small-cell lung cancer in Asia (IPASS). J Clin Oncol 29: 2866-2874, 2011.

20. Han JY, Park K, Kim SW, Lee DH, Kim HY, Kim HT, Ahn MJ, Yun T, Ahn JS, Suh C, et al: First-SIGNAL: First-line single-agent iressa versus gemcitabine and cisplatin trial in never-smokers with adenocarcinoma of the lung. J Clin Oncol 30: 1122-1128, 2012.

21. Wu YL, Zhou C, Hu CP, Feng J, Lu S, Huang Y, Li W, Hou M, Shi JH, Lee KY, et al: Afatinib versus cisplatin plus gemcitabine for first-line treatment of Asian patients with advanced non-small-cell lung cancer harbouring EGFR mutations (LUX-Lung 6): An open-label, randomised phase 3 trial. Lancet Oncol 15: 213-222, 2014. 
22. Yang JC, Wu YL, Schuler M, Sebastian M, Popat S, Yamamoto N Zhou C, Hu CP, O'Byrne K, Feng J, et al: Afatinib versus cisplatin-based chemotherapy for EGFR mutation-positive lung adenocarcinoma (LUX-Lung 3 and LUX-Lung 6): Analysis of overall survival data from two randomised, phase 3 trials. Lancet Oncol 16: 141-151, 2015.

23. Banno E, Togashi Y, Kobayashi Y, Hayashi H, Mitsudomi T and Nishio K: Afatinib is especially effective against non-small cell lung cancer carrying an EGFR exon 19 deletion. Anticancer Res 35: 2005-2008, 2015

24. Fukuhara T, Maemondo M, Inoue A, Kobayashi K, Sugawara S, Oizumi S, Isobe $\mathrm{H}$, Gemma A, Harada M, Yoshizawa $\mathrm{H}$, et al: Factors associated with a poor response to gefitinib in the NEJ002 study: Smoking and the L858R mutation. Lung Cancer 88: $181-186,2015$.
25. Zhang Y, Sheng J, Kang S, Fang W, Yan Y, Hu Z, Hong S, Wu X, Qin T, Liang W and Zhang L: Patients with exon 19 deletion were associated with longer progression-free survival compared to those with L858R mutation after first-line EGFR-TKIs for advanced non-small cell lung cancer: A meta-analysis. PLoS One 9: e107161, 2014.

26. Igawa S, Kasajima M, Ishihara M, Kimura M, Hiyoshi $Y$, Asakuma M, Otani S, Katono K, Sasaki J and Masuda N: Comparison of the efficacy of gefitinib in patients with non-small cell lung cancer according to the type ofepidermal growth factor receptor mutation. Oncology 87: 215-223, 2014. 